\title{
Same day discharge does not lead to worse outcomes in patients undergoing uncomplicated laparoscopic foregut surgery
}

\author{
M. Leah Brazer ${ }^{1} \mathbb{D} \cdot$ Dylan M. Russell $^{1} \cdot$ Scott H. Nguyen ${ }^{1} \cdot$ Christopher G. Yheulon $^{1}$
}

Received: 5 September 2021 / Accepted: 25 January 2022 / Published online: 14 February 2022

This is a U.S. government work and not under copyright protection in the U.S.; foreign copyright protection may apply 2022

\begin{abstract}
Introduction With the advancement of minimally invasive surgical techniques surgeons have moved away from elective open foregut surgeries. Despite studies demonstrating the safety of same day discharge in appropriate patient populations, ambulatory surgery has yet to be established as the practice norm for patients undergoing uncomplicated laparoscopic foregut surgery.

Methods The ACS-NSQIP database was queried from 2005 to 2018 for patients who had undergone elective and non-emergent laparoscopic Heller myotomy, fundoplication, and paraesophageal hernia repairs with and without mesh. The primary endpoints in this study included number and severity of complications as classified by the Clavien-Dindo Classification, readmission, and return to the operating room.

Results 6893 patients who met inclusion criteria were identified, 696 (10.1\%) of which were discharged on the day of surgery. Patients who were discharged on post-operative day one were matched at a 3:1 ratio producing 2088 comparisons. There was no difference in overall morbidity ( $p=0.264)$, readmission (OR $0.849,95 \%$ CI $0.522-1.419)$, or return to the operating room (OR 1.15, 95\% CI 0.531-2.761) between the two groups.

Conclusion Same day discharge for patients without life threatening comorbidities undergoing elective minimally invasive Heller myotomy, Nissen and Toupet fundoplication, and paraesophageal hernia repairs is safe and feasible.
\end{abstract}

Keywords Fundoplication · Paraesophageal $\cdot$ Esophagomyotomy $\cdot$ Heller $\cdot$ Hiatal $\cdot$ Foregut $\cdot$ Laparoscopic $\cdot$ Ambulatory

Minimally invasive surgery (MIS) has demonstrated clear benefit to patient outcomes in terms of decreasing post-operative pain and length of stay. Nowhere is this more apparent than in minimally invasive benign foregut surgery; early studies found that when compared to open surgery, patients who underwent laparoscopic surgery were discharged home more than 4 days earlier [1,2]. This reduction in hospital stay translates directly to significantly decreased health care costs.

Same day, day case, or ambulatory surgery are synonymous entities that emphasize minimal disruption of the patient's life with the patient discharging from the hospital the day of surgery [3]. The benefits of ambulatory surgery include increased patient comfort and satisfaction, decreased hospital costs, and optimized staff and resource utilization.

M. Leah Brazer

Miriam.1.brazer.mil@mail.mil

1 Department of Surgery, Tripler Army Medical Center, 1 Jarrett White Rd, Honolulu, HI 96859, USA
Multiple feasibility studies have been performed demonstrating its efficacy and safety in the appropriate patient population [4-7]. Despite this evidence, ambulatory surgery has yet to be established as the norm for benign foregut procedures. The purpose of this study is to better clarify the role of ambulatory surgery for well selected patients undergoing laparoscopic foregut procedures.

\section{Methods}

\section{Inclusion of patients}

The ACS-NSQIP database was queried from 2005 to 2018 for all adult patients undergoing laparoscopic foregut surgery using CPT codes. The CPT codes 43280, 43281, 43282 , 43279, correspond with laparoscopic fundoplication, laparoscopic paraesophageal hernia repair without mesh, laparoscopic paraesophageal hernia repair with mesh, and laparoscopic esophagomyotomy respectively. Patients 
were excluded from this study if they underwent emergent surgery, had an ASA classification of four or five, were preoperatively septic or on a ventilator, their operation was not performed by a general or cardiothoracic surgeon, or there were any concurrent non-endoscopic procedures. Records with incomplete demographic or outcomes data were also excluded. Analysis of data was performed with $\mathrm{R}$, an open source statistical computing software with the nsqipr package [8].

\section{Endpoints}

The primary endpoints were number and severity of complications as classified by the Clavien-Dindo classification system, readmission, and return to the operating room.

\section{Statistical analysis}

A 3:1 nearest-neighbor propensity-score match was conducted using the MatchIt package [9]. Patients discharged on post-operative day one versus those discharged on the day of surgery were matched on demographic data and pre- and intra-operative risk factors to achieve a standardized mean difference $<0.1$ for all measured covariates. The measured covariates and the pre- and post-match standardized mean difference are seen in Tables 1 and 2, respectively. Hypothesis tests of all outcomes were conducted using Fisher's exact test for count data with significance considered $p$ less than 0.05 .

\section{Results}

A total of 6893 patients with appropriate CPT codes and conforming to inclusion criteria were considered for the match. Prior to matching, 6197 and 696 patients were discharged on post-operative day one and the day of surgery, respectively. Significant differences in standardized mean difference existed for many of the measured covariates, including the type of surgery performed (fundoplication, Heller myotomy, paraesophageal hernia repair with mesh), age, sex, insulin use, smoking status, pre-operative dyspnea, use of anti-hypertensives, and ASA class (Table 1). After matching, a total of 2784 remained with 2088 and 696 patients discharged on post-operative day one and the

Table 1 Pre-match covariates

\begin{tabular}{llll}
\hline & POD 0 & POD 1 & SMD \\
& 696 & 6197 & \\
\hline Fundoplication (\%) & $302(43.4)$ & $2309(37.3)$ & 0.125 \\
Heller myotomy (\%) & $27(3.9)$ & $452(7.3)$ & 0.149 \\
PEHR with mesh (\%) & $94(13.5)$ & $1088(17.6)$ & 0.112 \\
PEHR with no mesh (\%) & $273(39.2)$ & $2348(37.9)$ & 0.027 \\
Age (mean (SD)) & $51.98(14.70)$ & $56.65(14.45)$ & 0.320 \\
Male (\%) & $295(42.4)$ & $2113(34.1)$ & 0.171 \\
BMI (mean (SD)) & $29.89(5.57)$ & $30.16(5.50)$ & 0.049 \\
Insulin use (\%) & $6(0.9)$ & $129(2.1)$ & 0.101 \\
Smokers (\%) & $111(15.9)$ & $693(11.2)$ & 0.140 \\
Pre-op dyspnea (\%) & $45(6.5)$ & $596(9.6)$ & 0.116 \\
History of COPD (\%) & $20(2.9)$ & $217(3.5)$ & 0.036 \\
History of CHF (\%) & $1(0.1)$ & $13(0.2)$ & 0.016 \\
Pre-operative anti-hypertensives (\%) & $235(33.8)$ & $2533(40.9)$ & 0.147 \\
Pre-operative dialysis (\%) & $0(0.0)$ & $2(0.0)$ & 0.025 \\
Disseminated cancer (\%) & $0(0.0)$ & $8(0.1)$ & 0.051 \\
Pre-operative wound infection (\%) & $2(0.3)$ & $6(0.1)$ & 0.044 \\
Pre-operative steroid use (\%) & $15(2.2)$ & $232(3.7)$ & 0.094 \\
Weight loss >10\% (\%) & $5(0.7)$ & $81(1.3)$ & 0.059 \\
Bleeding disorder (\%) & $7(1.0)$ & $64(1.0)$ & 0.003 \\
Pre-operative sepsis (\%) & $2(0.3)$ & $6(0.1)$ & 0.044 \\
ASA class (\%) & & & 0.252 \\
1 & $28(4.0)$ & $2431(39.2)$ & \\
2 & $473(68.0)$ & $0(0.0)$ & \\
3 & $195(28.0)$ & $109.73(51.19)$ & \\
4 & $0(0.0)$ & & \\
Operative time (minutes) (mean (SD)) & $89.48(48.59)$ & & \\
\hline & & & \\
\hline
\end{tabular}


Table 2 Post-match covariates

\begin{tabular}{|c|c|c|c|}
\hline & $\begin{array}{l}\text { POD } 0 \\
696\end{array}$ & $\begin{array}{l}\text { POD } 1 \\
2088\end{array}$ & SMD \\
\hline Fundoplication (\%) & $302(43.4)$ & $913(43.7)$ & 0.007 \\
\hline Heller myotomy (\%) & $27(3.9)$ & $91(4.4)$ & 0.024 \\
\hline PEHR with mesh (\%) & $94(13.5)$ & $274(13.1)$ & 0.011 \\
\hline PEHR with no mesh (\%) & $273(39.2)$ & $810(38.8)$ & 0.009 \\
\hline Age (mean (SD)) & $51.98(14.70)$ & $51.47(15.47)$ & 0.034 \\
\hline Male (\%) & $295(42.4)$ & $914(43.8)$ & 0.028 \\
\hline BMI (mean (SD)) & $29.89(5.57)$ & $29.99(5.47)$ & 0.018 \\
\hline Insulin use (\%) & $6(0.9)$ & $34(1.6)$ & 0.069 \\
\hline Smokers $(\%)$ & $111(15.9)$ & $330(15.8)$ & 0.004 \\
\hline Pre-op dyspnea (\%) & $45(6.5)$ & $159(7.6)$ & 0.045 \\
\hline History of COPD (\%) & $20(2.9)$ & $59(2.8)$ & 0.003 \\
\hline History of $\mathrm{CHF}(\%)$ & $1(0.1)$ & $3(0.1)$ & $<0.001$ \\
\hline Pre-operative anti-hypertensives (\%) & $235(33.8)$ & $693(33.2)$ & 0.012 \\
\hline Pre-operative dialysis (\%) & $0(0.0)$ & $2(0.1)$ & 0.044 \\
\hline Disseminated cancer (\%) & $0(0.0)$ & $2(0.1)$ & 0.044 \\
\hline Pre-operative wound infection (\%) & $2(0.3)$ & $2(0.1)$ & 0.044 \\
\hline Pre-operative steroid use (\%) & $15(2.2)$ & $69(3.3)$ & 0.071 \\
\hline Weight loss >10\% (\%) & $5(0.7)$ & $27(1.3)$ & 0.058 \\
\hline Bleeding disorder $(\%)$ & $7(1.0)$ & $16(0.8)$ & 0.026 \\
\hline Pre-operative sepsis (\%) & $2(0.3)$ & $3(0.1)$ & 0.031 \\
\hline ASA class $(\%)$ & & & 0.034 \\
\hline 1 & $28(4.0)$ & $76(3.6)$ & \\
\hline 2 & $473(68.0)$ & $1450(69.4)$ & \\
\hline 3 & $195(28.0)$ & $562(26.9)$ & \\
\hline 4 & $0(0.0)$ & $0(0.0)$ & \\
\hline Operative time (mean (SD)) & $89.48(48.59)$ & $91.56(46.41)$ & 0.044 \\
\hline
\end{tabular}

Table 3 Outcomes

\begin{tabular}{llll}
\hline & $\begin{array}{l}\text { POD 0 } \\
696\end{array}$ & $\begin{array}{l}\text { POD 1 } \\
2088\end{array}$ & $p$ \\
\hline $\begin{array}{llll}\text { Clavien-dindo class (\%) } \\
\text { None }\end{array}$ & $676(97.1)$ & $2018(96.6)$ & 0.261 \\
Minor & $7(1.0)$ & $39(1.9)$ & \\
Major & $12(1.7)$ & $30(1.4)$ & \\
Death & $1(0.1)$ & $1(0.0)$ & \\
Readmission (\%) & $25(3.6)$ & $70(3.4)$ & 0.809 \\
Return to OR (\%) & $9(1.3)$ & $30(1.4)$ & 0.855 \\
\hline
\end{tabular}

day of surgery, respectively. A standardized mean difference of less than 0.1 was achieved for all measured covariates, suggesting a suitable match (Table 2).

After matching, no significant difference was demonstrated in Clavien-Dindo outcomes between the two groups $(p=0.264)$, rates of readmission (OR 0.849 , $p=0.534)$, or rates of return to the operating room (OR $1.15, p=0.855$ ) (Table 3 ).

\section{Discussion}

Using the power of ACS-NSQIP database, this is now one of the largest retrospective analyses examining the role of ambulatory surgery for patients undergoing laparoscopic foregut surgery. Uncomplicated laparoscopic foregut surgery is commonly performed with a hospital stay of at least 1 day $[10,11]$. However, in this study, we found that well selected patients undergoing uncomplicated laparoscopic foregut surgery had no differences in objective perioperative outcomes regardless if they were discharged on the day of surgery or post-operative day one.

Same day surgery for laparoscopic fundoplication has been studied more extensively than either laparoscopic paraesophageal hernia repair or laparoscopic Heller myotomy with the publication of various sized retrospective and prospective cohort studies starting in the early 2000s. A 
systematic review of the data was published in 2011 that included 13 different studies, 10 of which looked at planned same day discharge (792 patients) and 3 on planned $23 \mathrm{~h}$ discharge (583 patients) [12]. In contrast, there are only a handful of smaller cohort studies looking the safety and feasibility of same day discharge for laparoscopic Heller myotomy as well as paraesophageal hernia repair in select populations $[6,10,11,13,14]$. Our study population was a group of patients that we deemed low risk for ambulatory surgery. While ambulatory surgery for laparoscopic foregut surgery may not be possible in all patients, we aimed to identify a population who can safely forego postoperative hospitalization.

As Enhance Recovery After Surgery (ERAS) protocols have become more ubiquitous and effective with the advent of minimally invasive surgery and patient centered health care models, there is continued expansion of the application of ERAS protocols to different types of surgeries. Multimodal pain control, early PO intake, and aggressive control and mitigation of post-operative nausea and vomiting (PONV) used in ERAS protocols can be applied to laparoscopic hiatal surgery in order to help facilitate same day discharge. Previous studies that have looked at why patients undergoing laparoscopic hiatal surgery who were planned to be discharged later that day do not end up going home and found that PONV, poorly controlled pain secondary to both incisional pain and diaphragmatic irritation from insufflation, and patient preference to be the main reasons for overnight admission $[10,11]$. There are known anesthetic techniques that can be implemented during the perioperative period to reduce PONV. Poor post-operative pain control can be mitigated by providing patients with realistic expectations for their post-operative pain, using multimodal pain control, and reducing insufflation pressures intraoperatively. Patient preference for overnight admission is difficult to control, however, the data show that thorough pre-operative assessment of patient comorbidities, support systems, health literacy, and mental health improves post-operative recovery after same day surgery as well as increasing patient satisfaction [15]. While there will always be patients who require overnight admission for uncomplicated foregut surgery, the paradigm can shift to most patients successfully undergoing same day surgery with careful pre-operative planning and patient education.

\section{Relevance to the Covid-19 pandemic}

With the rise of the global COVID-19 pandemic, it is more important than ever to safely minimize length of hospital stay in order to decrease the utilization of valuable healthcare resources and reduce nosocomial transmission of SARS-CoV-2. Earlier in the pandemic elective surgeries were postponed across most hospitals in the USA in order to free up hospital beds and supplies to treat COVID-19 patients. However, as the pandemic progressed, operating rooms returned to full capacity, performing elective cases and surgeons of all specialties began developing perioperative protocols aimed to safely turn operations that have traditionally required overnight hospitalization into day cases, specifically same day breast reconstruction and same day robotic urologic surgery $[16,17]$. Same day discharge of patients undergoing uncomplicated foregut surgery appears to be a safe option and viable option in general but is even more critical during an ongoing pandemic resulting in scarcity hospital beds and healthcare resources.

\section{Limitations}

The limitations of this study are due to its retrospective nature. In spite of our efforts to create a study with a large sample size and control for selection bias, all retrospective studies can have the introduction of bias due to inaccurate or missing information in the database. It is also impossible to determine which if any patients discharged on post-operative day one were intended to have had a same day surgery and were hospitalized an extra day for clinical reasons vs. those whose surgeons' practice it is to discharge patients on postoperative day one. There are other factors that likely affect when patients are discharged such as size of a paraesophageal hernia or difficulty and complexity of an operation. Unfortunately these factors are not addressed by the NSQIP database.

The decision to include laparoscopic Heller myotomy in this study was deliberate. Although the majority of laparoscopic Heller myotomies in this study are not performed on an ambulatory basis $(27 / 479,5.6 \%)$, this is not much different from the frequency of the other included operations in this study (Fundoplication 11.6\%, PEHR with mesh 8.0\%, PEHR without mesh $10.4 \%$ ). We believe the main purpose of this paper is to define which cases can be safely performed in an ambulatory setting as this influences practice patterns. This is especially true in an environment like the COVID-19 pandemic where inpatient beds can be the rate limiting step. The findings of our paper add to the growing literature supporting ambulatory Laparoscopic Heller Myotomy for well selected patients $[13,18]$.

Lastly, while we were able to determine certain shortterm outcomes using the ACS-NSQIP database, other important outcomes such as recurrence rates of paraesophageal hernia repairs and recurrence of symptoms in patients who underwent fundoplication or Heller myotomy are unknown. 


\section{Future considerations}

The safety and feasibility of same day discharge for uncomplicated elective hiatal surgery could be further elucidated with a randomized control trial which would allow for less risk of bias. Ongoing work to expand the patient population who can be discharge home safely the day of surgery is needed.

\section{Conclusion}

Although most patients who undergo minimally invasive Heller myotomy, Nissen and Toupet fundoplication, and paraesophageal hernia repairs are discharged on post-operative day one, discharge on the day of surgery appears to be safe in patients without life threatening comorbidities and whose surgery was uncomplicated. There are no associated increased rates of complication, readmission, or return to the operating room compared to patients discharged on postoperative day one. Discharging patients home on the day of surgery will free up healthcare resources and personnel allowing for more efficient utilization of resources.

\section{Disclosures}

Drs. Brazer, Russell, Nguyen, and Yheulon have no conflicts of interest or any relevant financial ties to disclose.

Acknowledgements The authors received no funding or other support for the creation of this manuscript to include the following organizations: National Institutes of Health (NIH); Wellcome Trust; Howard Hughes Medical Institute (HHMI); and other(s).

\section{References}

1. Farahmand M, Soot SJ, Rand-luby L, Deveney CW, Sheppard BC (1998) Comparison of outcomes of open versus laparoscopic nissen fundoplication performed in a single practice. Am J Surg 175:371-374

2. Nguyen NT, Christie C, Masoomi H, Matin T, Laugenour K, Hohmann S (2011) Utilization and outcomes of laparoscopic versus open paraesophageal hernia repair. Am Surg 77:1353-1357

3. Trondsen E, Mjåland O, Raeder J, Buanes T (2000) Day-case laparoscopic fundoplication for gastro-oesophageal reflux disease. Br J Surg 87:1708-1711
4. Bharatam KK, Raj R, Subramanian JB, Vasudevan A, Bodduluri S, Sriraman KB, Abineshwar NJ (2015) Laparoscopic nissen rossetti fundoplication: possibility towards day care anti-reflux surgeries. Ann Med Surg 4:384-387

5. Gronnier C, Desbeaux A, Piessen G, Boutillier J, Ruolt N, Triboulet JP, Mariette C (2014) Day-case versus inpatient laparoscopic fundoplication: outcomes, quality of life and cost-analysis. Surg Endosc 28:2159-2166

6. Molina JC, Misariu AM, Nicolau I, Spicer J, Mulder D, Ferri LE, Mueller CL (2018) Same day discharge for benign laparoscopic hiatal surgery: a feasibility analysis. Surg Endosc 32:937-944

7. Victorzon M, Tolonen P, Vuorialho T (2006) Laparoscopic floppy nissen fundoplication for gastro-oesophageal reflux disease is feasible as a day-case procedure. Scand J Surg 95:162-165

8. Russell D (2020) Easy interface with the NSQIP database using nsqipr. Available at: https://github.com/dylanrussellmd/ nsqipr. Accessed 12 Nov 2020

9. Ho DE, King G, Stuart EA, Imai K (2011) MatchIt: nonparametric preprocessing for parametric causal inference. J Stat Softw 42:1-28

10. Gutierrez R, Neill CO, Khanna A, Miller A, Banki F (2020) Laparoscopic hiatal hernia repair as same day surgery: feasibility, short-term outcomes and costs. Am J Surg 220:1438-1444

11. Kaushik C, Milhoan M, Khanna A, Miller A, Chawla M, Miller CC, Banki F (2019) Laparoscopic heller myotomy and dor fundoplication in the same day surgery setting with a trained team and an enhanced recovery protocol. Surg Open Sci 1:64-68

12. Thomas H, Agrawal S (2011) Systematic review of day-case laparoscopic fundoplication. J Laparoendosc Adv Surg Tech 21:781-788

13. Agrawal S, Super P (2008) Laparoscopic heller myotomy for achalasia: changing trend toward "true" day-case procedure. J Laparoendosc Adv Surg Tech 18:785-788

14. Desbeaux A, Gronnier C, Piessen G, Vanderbeken M, Ruolt N, Triboulet JP, Mariette C (2017) Same-day discharge in benign esophageal surgery: a prospective cohort study. Dis Esophagus $30: 1-7$

15. Jaensson M, Dahlberg K, Nilsson U (2019) Factors influencing day surgery patients' quality of postoperative recovery and satisfaction with recovery: a narrative review. Perioper Med 8:3

16. Abaza R, Kogan P, Martinez O (2021) Impact of the COVID-19 crisis on same-day discharge after robotic urologic surgery. Urology 49:40-45

17. Specht M, Sobti N, Rosado N, Tomczyk E, Abbate O, Ellis D, Liao EC (2020) High-efficiency same-day approach to breast reconstruction during the COVID-19 crisis. Breast Cancer Res Treat 182:679-688

18. Naumann DN, Zaman S, Daskalakis M, Naumann DN, Zaman S, Daskalakis M, Nijjar R, Richardson M, Super P, Singhal R (2016) Day surgery for achalasia cardia: time for consensus? Ann R Coll Surg Engl 98:150-154

Publisher's Note Springer Nature remains neutral with regard to jurisdictional claims in published maps and institutional affiliations. 